\title{
Unidirectional sub-100-ps magnetic vortex core reversal
}

\author{
Matthias Noske, ${ }^{1, *}$ Ajay Gangwar, ${ }^{1,2}$ Hermann Stoll, ${ }^{1}$ Matthias Kammerer, ${ }^{1}$ Markus Sproll,,${ }^{1}$ Georg Dieterle, ${ }^{1}$ \\ Markus Weigand, ${ }^{1}$ Manfred Fähnle, ${ }^{1}$ Georg Woltersdorf, ${ }^{3}$ Christian H. Back, ${ }^{2}$ and Gisela Schütz ${ }^{1}$ \\ ${ }^{1}$ Max Planck Institute for Intelligent Systems, Heisenbergstraße 3, 70569 Stuttgart, Germany \\ ${ }^{2}$ University of Regensburg, Department of Physics, Universitätsstraße 31, 93053 Regensburg, Germany \\ ${ }^{3}$ University of Halle, Department of Physics, Von-Danckelmann-Platz 3, 06120 Halle, Germany \\ (Received 21 May 2014; revised manuscript received 1 August 2014; published 19 September 2014)
}

\begin{abstract}
We experimentally demonstrate that unidirectional reversal of the magnetic vortex core polarity is possible by excitation with sub-100-ps-long orthogonal monopolar magnetic pulse sequences in a wide range of pulse lengths and amplitudes. The application of such short digital pulses is a favorable excitation scheme for technological applications. Measured phase diagrams of this unidirectional, spin-wave mediated vortex core reversal are in good qualitative agreement with phase diagrams obtained from micromagnetic simulations. The time dependence of the reversal process, observed by time-resolved scanning transmission x-ray microscopy indicates a switching time of $100 \mathrm{ps}$ and fits well with our simulations. The origin of the asymmetric response to clockwise and counterclockwise excitation which is a prerequisite for reliable unidirectional switching is discussed, based on the gyromode-spin-wave coupling. Situations are found in which a three-dimensional dynamics is important, because a vortex-antivortex pair starts to form close to the core of the original vortex in the lower part of the disk without completing the formation across the whole thickness so that it dissolves later on and does not lead to switching of the original vortex core.
\end{abstract}

DOI: 10.1103/PhysRevB.90.104415

PACS number(s): 75.30.Ds, 75.75.-c, 75.78.Cd

\section{INTRODUCTION}

In thin soft magnetic layers with thicknesses of a few tens of nanometers and lateral dimensions ranging from $100 \mathrm{~nm}$ to $10 \mu \mathrm{m}$, the vortex structure is the ground state showing an in-plane curling magnetization and a perpendicularly magnetized core at the center with a size of about 10-20 nm [1-3]. In spite of its high stability with respect to static external magnetic fields the vortex core can be switched dynamically with low-power sine or pulsed magnetic fields or spin-polarized currents [4-7]. This was achieved by resonantly exciting the gyrotropic mode with its eigenfrequency typically in the range from $100 \mathrm{MHz}$ to $1 \mathrm{GHz}$. By applying rotating in-plane fields this reversal occurs in a unidirectional manner [8-12] since the exciting field couples only to the vortex gyrotropic mode if the sense of rotation of the excitation corresponds to the sense of the vortex gyrotropic mode, which is determined by the core polarity. Due to these phenomena the vortex core has been discussed as an extremely stable bit element in digital magnetic random access storage media providing a low-power [7] and selectively addressable switching mechanism with a speed in the range of nanoseconds [9]. At much higher (gigahertz) frequencies vortex structures possess spin-wave eigenmodes arising from the magnetostatic interaction. Spinwave mediated vortex core reversal was demonstrated experimentally with excitation of multi-GHz rotating field bursts [13]. Hereby unidirectional vortex core reversal was achieved and corresponding micromagnetic simulations indicated a

\footnotetext{
*noske@is.mpg.de

Published by the American Physical Society under the terms of the Creative Commons Attribution 3.0 License. Further distribution of this work must maintain attribution to the author(s) and the published article's title, journal citation, and DOI.
}

switching time slightly above 200 ps [14,15] when using one-period bursts.

Here we explore, by systematic experimental studies and micromagnetic simulations, how to speed up unidirectional vortex core reversal by excitation with a sequence of two orthogonal monopolar magnetic pulses, less than 100 ps in total duration. The fact that digital pulses can be used for fast spin-wave mediated switching instead of rotating field bursts is an attractive aspect for potential technological applications. The phase diagram of vortex core reversal is measured, i.e., the dependence of switching on pulse amplitudes and their durations. A region of unidirectionality is found to be rather large and thus robust against sample dimension variations. The finding that the region of unidirectionality is larger than the corresponding region for rotating field bursts is a further important result. Time-resolved x-ray microscopy image sequences show the dynamics of the switching process, in excellent agreement with simulations. The measurements indicate a switching time below 100 ps which is in accordance with our simulations. Furthermore, the switching time of unidirectional vortex core reversal by pulsed excitation is simulated in samples of different sizes and the influence of material parameters is studied indicating a lower universal limit of about 70 ps for the switching time. Coupling between spin waves and the vortex gyromode is identified as the origin of the asymmetric response of the magnetization to clockwise $(\mathrm{CW})$ or counterclockwise $(\mathrm{CCW})$ excitation which is responsible for the unidirectionality in the vortex core reversal by this broadband orthogonal short pulse excitation.

\section{METHODS}

\section{A. Experiment}

Permalloy $\left(\mathrm{Ni}_{80} \mathrm{Fe}_{20}, 50 \mathrm{~nm}\right.$ thick) disks are thermally evaporated on top of crosslike copper $(\mathrm{Cu}, 150 \mathrm{~nm}$ thick) 

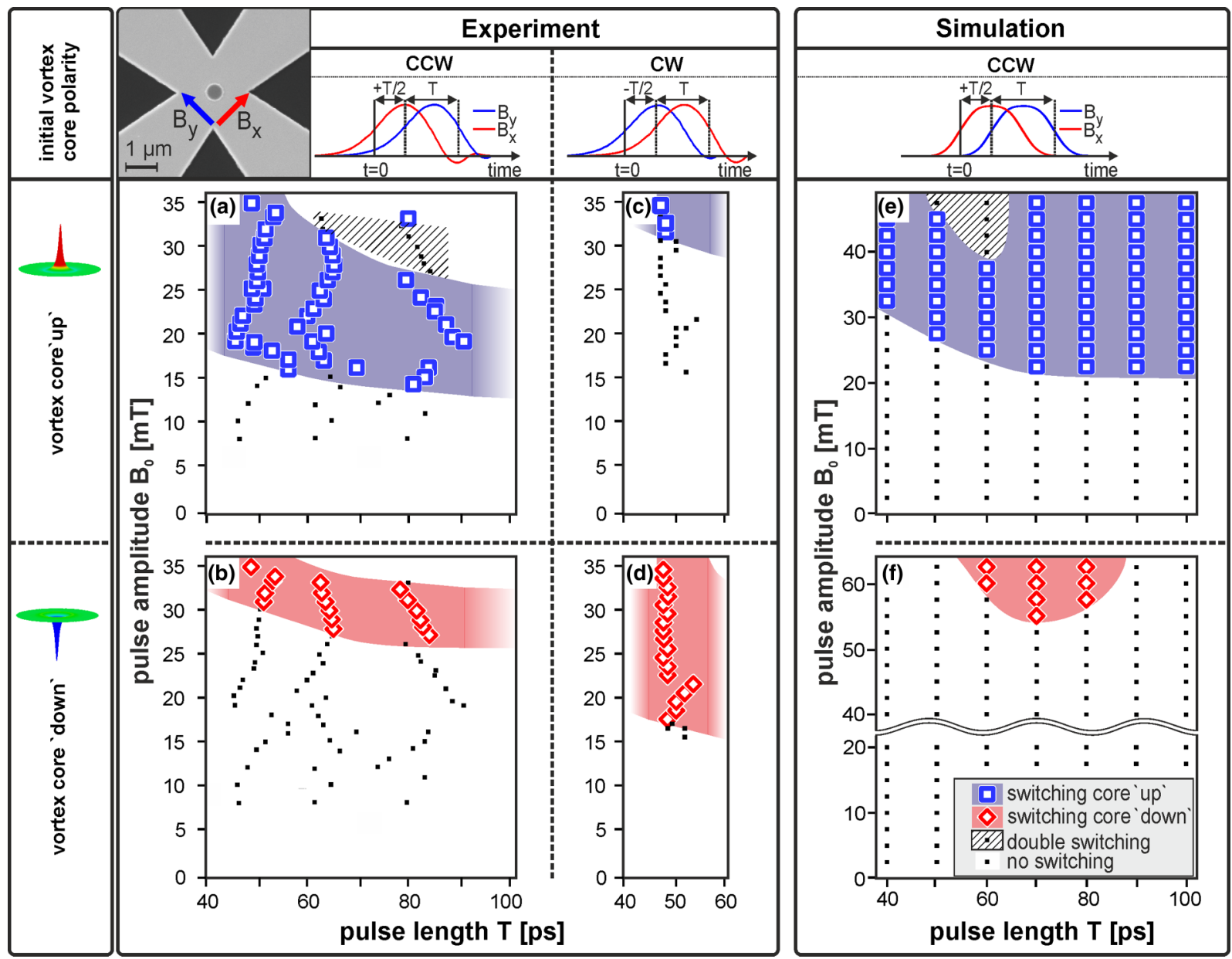

FIG. 1. (Color) Phase diagrams for vortex core reversal by pulsed excitation obtained experimentally [sample A: (a)-(d)] and by micromagnetic simulations (e), (f). A scanning electron microscope image of the crossed strip lines with the Permalloy disk is shown at the top. The arrows indicate the directions of the magnetic field pulses that produce the $\mathrm{CCW} / \mathrm{CW}$ excitation sequence. Unidirectional reversal is found in a broad region, where CCW excitation switches a vortex with core up (a), (e) but not a vortex with core down (b), (f). For CW excitation, this behavior is inverted (c), (d) as is expected for symmetry reasons.

strip lines with a width of $1.6 \mu \mathrm{m}$ (see top of Fig. 1). The results shown in Fig. 1 were obtained on a single vortex structure (sample A, diameter $490 \mathrm{~nm}$ ); the measurements displayed in Fig. 2 were performed on a different vortex structure (sample B, diameter $500 \mathrm{~nm}$ ). To avoid oxidation of the Permalloy, the disks are capped with a 4-nm Al layer. The samples are structured in multiple steps on top of silicon nitride membranes using a combination of optical and electron-beam lithography and lift-off processes. By sending current pulses through the crossed strip lines, magnetic field pulses up to field strengths of $35 \mathrm{mT}$ can be generated to excite the Permalloy sample. A sampling oscilloscope with a bandwidth of $50 \mathrm{GHz}$ is used to monitor the exciting signals allowing the calculation of the magnetic field strength at the location of the sample [10]. The vortex structures are investigated by scanning transmission X-ray microscopy at the MAXYMUS end station at BESSY II, Berlin, which provides a lateral resolution of $25 \mathrm{~nm}$ and high magnetic contrast based on the X-ray magnetic circular dichroism effect [16] at the $\mathrm{Ni} L_{3}$ edge. A sophisticated lock-in-like data acquisition scheme allows low-noise time-resolved pump-and-probe measurements of the magnetization dynamics of the vortex structure with a temporal resolution down to $45 \mathrm{ps}$. The complete pump-and-probe cycle for the measurement of the reversal process shown in Fig. 2 consists of the CCW pulse sequence shown in the figure and an additional $\mathrm{CW}$ pulse sequence at $t=6.5 \mathrm{~ns}$ that resets the vortex core to its initial "up" polarity (not shown in Fig. 2). The complete measurement consists of 731 images at different time steps. Each image is divided by the average of all images to eliminate static image contributions. The results are normalized images as shown in Fig. 2. The stroboscopic experiment is repeated with a frequency of $75 \mathrm{MHz}$ over more than $10^{11}$ cycles to obtain the required signal to noise ratio. The excitation consists of two orthogonal monopolar magnetic in-plane field pulses with pulse amplitude $B_{0}$, pulse length $T$, and a delay of $\frac{1}{2} T$ between the two pulses (see top left of Fig. 1). The resulting total magnetic field is $\mathbf{B}(t)=\left[B_{0} p\left(t-\frac{1}{2} T, T\right), B_{0} p(t, T), 0\right]$ corresponding to $\mathrm{CW}$ excitation and $\mathbf{B}(t)=\left[B_{0} p(t, T), B_{0} p\left(t-\frac{1}{2} T, T\right), 0\right]$ for CCW excitation, where $p(t, \mathrm{~T})$ describes the pulse shape. In the simulations, $p(t, \mathrm{~T})$ is approximated by

$$
p(t, T)=\frac{1}{4}[\operatorname{erf}(t k)+1]\{1-\operatorname{erf}[(t-T) k]\},
$$




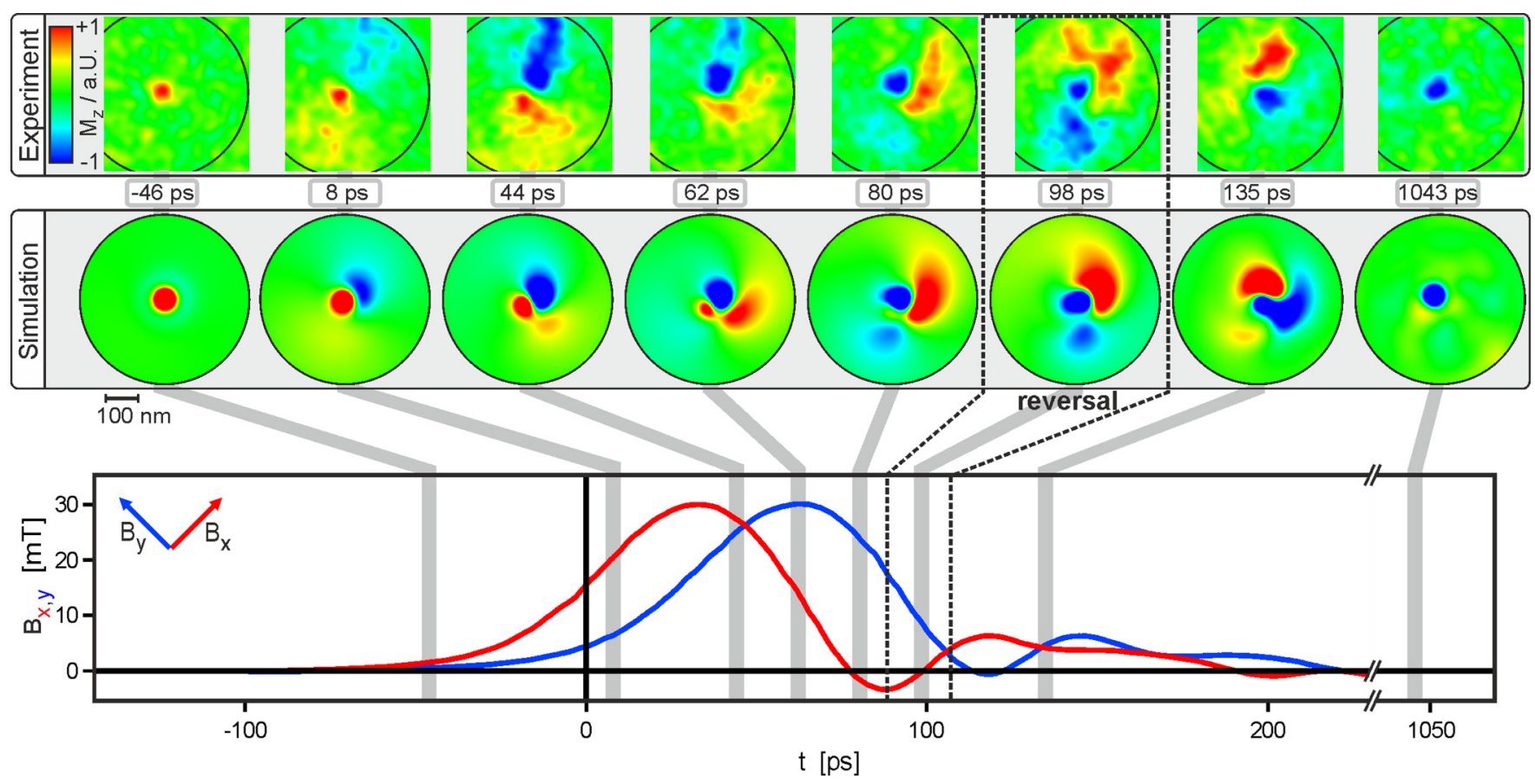

FIG. 2. (Color) Time-resolved imaging of the VC reversal process by the excitation with orthogonal field pulses of $30 \mathrm{mT}$ strength, pulse width of $60 \mathrm{ps}$ and a delay of $30 \mathrm{ps}$ (shown in the lower row). The top row shows the dynamic response of the out-of-plane magnetization measured with time-resolved STXM (sample B). The middle row shows the $z$ component obtained from the corresponding micromagnetic simulation averaged over the thickness and convolved with the experimental temporal and lateral resolution.

where erf is the error function and $k$ defines the rise time of the pulses (see top right of Fig. 1). We define $t=0 \mathrm{ps}$ when the first pulse reaches $50 \%$ of its full amplitude as the start of the excitation and $t=3 / 2 T$ when the second pulse falls below $50 \%$ of its full amplitude as the end of the excitation. Correspondingly the total excitation time is $3 / 2 T$. To determine the switching phase diagram the core polarity of the vortex structures is measured before and after the pulse excitation.

\section{B. Micromagnetic simulations}

Three-dimensional micromagnetic simulations are performed using the object-oriented micromagnetic framework (OOMMF) [17]. Unless otherwise stated, disk-shaped platelets with a diameter of $500 \mathrm{~nm}$ and a thickness of $50 \mathrm{~nm}$ with cubic simulation cells of $(3.125 \mathrm{~nm})^{3}$ are used. The damping constant $\alpha=0.0077$ and saturation magnetization $M_{S}=830 \mathrm{kA} / \mathrm{m}$ are derived from the damped vortex core gyration after exciting sample B with a magnetic monopolar pulse and measured using time-resolved scanning transmission $\mathrm{x}$-ray microscopy (STXM). Typical values for Permalloy for the exchange constant $\left(A_{\mathrm{Ex}}=13 \times 10^{-12} \mathrm{~J} / \mathrm{m}\right)$ and the gyromagnetic ratio $\left(\gamma=2.21 \times 10^{5} \mathrm{~m} / \mathrm{As}\right)$ are used. The anisotropy constant is set to zero $\left(K_{1}=0\right)$. The position of the vortex core in Figs. $4(c)$ and 4(d) is tracked by fitting a Gaussian to the $z$ component of the magnetization.

\section{RESULTS}

The experimentally monitored switching behavior for both vortex core polarities is presented in Figs. 1(a)-1(d). The pulse length $T$ is varied from 45 to 90 ps and the delay between the two pulses is always tuned to $\frac{1}{2} T$. This results in a total excitation time ranging from 67 to 135 ps. For a CCW sense of rotation [Figs. 1(a) and 1(b)] the pulse $B_{x}$ in the $x$ direction starts before the pulse $B_{y}$ in the $y$ direction. For this sense of rotation, it is found that the switching threshold for an initial vortex core "down" is nearly twice as high as for an initial vortex core up. This asymmetry in switching threshold is nearly independent of the pulse length and proves the unidirectionality of the process for pulse amplitudes $B_{0}$ ranging from 15 to $30 \mathrm{mT}$. Due to symmetry reasons, a vortex core up (down) excited with a $\mathrm{CW}$ sequence is expected to have the same switching behavior as a vortex core down (up) excited by a CCW sequence. This is verified for the shortest pulse lengths by inverting the timing of the pulse sequence so that $B_{y}$ started before $B_{x}$ corresponding to a clockwise excitation. Within the accuracy of the measurements the switching threshold found for core up at CW excitation [Fig. 1(c)] is the same as for core down at CCW excitation [Fig. 1(b)] and the threshold for core down at CW excitation [Fig. 1(d)] is the same as for core up at CCW excitation [Fig. 1(a)]. Due to this agreement in combination with the symmetry argument this test is omitted for longer pulse lengths. The measurements shown in Fig. 1 further demonstrate that highly reliable and reproducible unidirectional switching is possible since in the connected areas in the phase diagram representing switching (nonswitching) events not a single nonswitching (switching) event is found. Additionally, the pulse lengths of only 45 ps and total excitation times of $67 \mathrm{ps}$ are the shortest values shown for unidirectional vortex core reversal.

The results of the three-dimensional micromagnetic simulations are shown in Figs. 1(e) and 1(f) and demonstrate a good qualitative agreement with the experimentally observed asymmetry in switching thresholds. For high pulse amplitudes 
a region exists in the phase diagram [Fig. 1(a), shaded area] where the vortex core polarity after excitation is the same as before. Analyzing the corresponding simulations of Fig. 1(e), this is explained by double switching events. For further increased amplitudes even triple switching is observed. It has to be pointed out that the switching thresholds in our experiments are found to be systematically lower by about $33 \%$ compared to the simulations. This tendency, which has been observed before in the case of core reversal by static out-of-plane fields [18], seems to be a systematic phenomenon. It may partly be explained by sample defects acting as nucleation centers for the reversal process and by thermal excitations, which are not accounted for in the simulations. Additionally, in micromagnetic simulations the treatment of a Bloch point which is present during the reversal process is problematic and can increase the switching threshold as discussed in detail by Thiaville et al. in Ref. [18]. It might be of considerable technological interest that the experimental switching thresholds are systemically lower than expected from the simulations as the required fields can be realized more easily.

\section{A. Spin-wave mediated vortex core reversal}

To obtain more insight into the dynamics of the reversal process the out-of-plane component of the magnetization during the switching process is imaged by stroboscopic time-resolved scanning transmission $\mathrm{x}$-ray microscopy and compared with the result from a corresponding micromagnetic simulation (Fig. 2). The excitation pulses have a length of $T=60 \mathrm{ps}$, a pulse amplitude of $B=30 \mathrm{mT}$, and a delay of $30 \mathrm{ps}$ between the two pulses corresponding to a CCW excitation. The experimental excitation is shown in Fig. 2 and is also used for the simulation. Note that this experimental pulse shape deviates slightly from the ideal shape given by $\mathbf{B}(t)=\left[B p(t, T), B p\left(t-\frac{1}{2} T, T\right), 0\right]$. However, no significant deviations are found when repeating the simulation with the ideal shape. The simulation is convolved with the experimental lateral and temporal resolution. Measurements and simulations show excellent agreement. The vortex core initially points up (red dot). During the rising edge of the first pulse, a bipolar structure forms similar to an $n=1, m=+1$ spin wave [13]. The counterclockwise rotation of this structure follows the rotational sense of the excitation field. From $t \approx 45 \mathrm{ps}$ to $t \approx 85 \mathrm{ps}$ the downwards oriented magnetization (blue) becomes localized close to the position of the original vortex core forming a "dip" while the core smears out and its contrast reduces. The latter can be explained by a compression of the vortex core in combination with tilting of the core from the $z$ direction. The measurement indicates core reversal at about $t=100 \mathrm{ps}$. From the (unconvolved) simulations it can be deduced that at $t=85 \mathrm{ps}$ the switching process begins and is completed at $t=106 \mathrm{ps}$ when the original vortex core up has switched to a vortex core pointing down (blue spot) in all discretization layers of the three-dimensional simulation. To characterize the speed of the reversal we define a switching time $\theta$ which measures the time from the start of the excitation until the core is completely reversed. In this case the switching time $\theta$ is $106 \mathrm{ps}$.

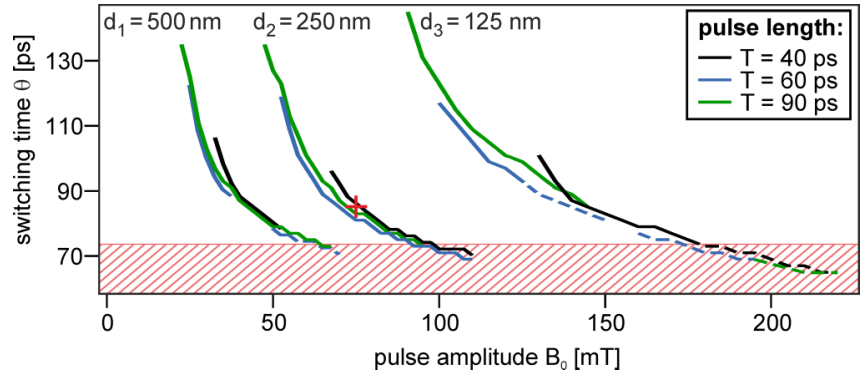

FIG. 3. (Color) Switching time as a function of pulse amplitude $B$ derived from micromagnetic simulations. Results for three samples of different diameter $(d=500 \mathrm{~nm}, d=250 \mathrm{~nm}, d=125 \mathrm{~nm})$ and three different pulse lengths $T$ are shown. Dashed lines indicate where the unidirectionality is lost since switching occurs for both vortex core polarities. The switching time only slightly depends on the pulse length. For all three samples unidirectional switching within $70-75 \mathrm{ps}$ is possible.

\section{B. Speed of the reversal process}

When thinking about a possible application of the vortex core as fast storage element it might be of interest how this switching time $\theta$ depends on sample parameters and the excitation. Additionally, to achieve higher storage densities smaller elements are favorable. Therefore the switching times as a function of pulse amplitude and pulse length $(T=40 \mathrm{ps}$, $T=60 \mathrm{ps}, T=90 \mathrm{ps}$ ) are deduced from the micromagnetic simulations shown in Fig. 1 (for a diameter $d_{1}=500 \mathrm{~nm}$ ) and for two additional Permalloy platelets with smaller diameter $\left(d_{2}=250 \mathrm{~nm}, d_{3}=125 \mathrm{~nm}\right)$, all with a thickness of $50 \mathrm{~nm}$. The results are shown in Fig. 3. The switching time strongly depends on the pulse amplitude but surprisingly is nearly independent of the pulse length. Furthermore, smaller disks below diameters of $500 \mathrm{~nm}$ do not allow shorter switching times. When scaling the pulse amplitudes with the inverse diameter (500-nm disk: $B^{\prime}=d_{1} / d_{1} B_{0} ; 250$-nm disk: $B^{\prime}=$ $d_{2} / d_{1} B_{0} ; 125-\mathrm{nm}$ disk: $\left.B^{\prime}=d_{3} / d_{1} B_{0}\right)$, the switching time shows similar dependence of this scaled pulse amplitude $B^{\prime}$ for all three samples. Unidirectional core reversal is possible with switching times as short as 75 ps in all cases. Higher pulse amplitudes would allow even shorter switching times; however, the unidirectionality is lost since switching occurs for both core polarities (indicated by the dashed lines). Especially in the smallest sample, the pulse length has to be chosen appropriately since there the region of unidirectionality depends most strongly on the pulse length. To estimate the influence of the remaining parameters (sample thickness, material properties, cell size of the simulation), each of these parameters is varied separately starting from the simulation marked by the red cross in Fig. $3\left(d_{2}=250 \mathrm{~nm}, B=75 \mathrm{mT}\right.$, $T=40 \mathrm{ps}$, switching time $\theta=86 \mathrm{ps}$ ). It is found that the saturation magnetization has the strongest influence on the switching time $\theta$ which can be easily explained. A reduction from $M_{S}=830 \mathrm{kA} / \mathrm{m}$ to $M_{S}=660 \mathrm{kA} / \mathrm{m}$ increases $\theta$ from 86 to 100 ps due to the fact that less energy is coupled into the system by the excitation pulses leading to a later start of the reversal process. A reduction of the thickness from 50 to $41 \mathrm{~nm}$ reduces the switching time to $80 \mathrm{ps}$ while increasing the thickness to $59 \mathrm{~nm}$ increases the switching time to $100 \mathrm{ps}$. 
Here for the three different thicknesses the reversal process starts at $t=70 \mathrm{ps}$, but the actual reversal takes longer for thicker samples. Varying the damping constant in the range from $\alpha=0.001$ to $\alpha=0.01$ or the exchange constant in the range from $A_{\mathrm{Ex}}=11 \times 10^{-12} \mathrm{~J} / \mathrm{m}$ to $A_{\mathrm{Ex}}=15 \times 10^{-12} \mathrm{~J} / \mathrm{m}$ has no detectable influence on the switching time. Reducing the cell size of the simulation from $(3.215 \mathrm{~nm})^{3}$ to $(2 \mathrm{~nm})^{3}$ increases the switching time by 4 ps which is probably related to the Bloch point present during the reversal process $[18,19]$.

\section{DISCUSSION}

In the final part we discuss the physical origin of the unidirectionality of vortex core reversal with very short orthogonal magnetic pulses. It differs fundamentally from the excitation with a rotating magnetic field with a well-defined frequency [13]. There the frequency can be tuned to a specific $\mathrm{CW}$ or CCW spin-wave mode eigenfrequency and excitation only occurs if both the frequencies and the senses of rotation of the external field and this specific spin-wave mode are the same. Only if this is the case, energy is resonantly coupled into the system and vortex core reversal occurs. In contrast, the very short pulses used in the present paper show a several $\mathrm{GHz}$ broad frequency spectrum and thus frequency tuning to a specific $\mathrm{CW}$ or CCW vortex core eigenmode fails. Nevertheless, a unidirectional vortex core reversal is found for such a short and broadband excitation (see Fig. 1), in spite of the fact that our micromagnetic simulations reveal that the energy coupled into the system is nearly the same for $\mathrm{CW}$ and $\mathrm{CCW}$ excitation.

We suggest assigning the physical origin for this $\mathrm{CW}-\mathrm{CCW}$ asymmetry for short pulse excitation to a coupling of the spin waves with the gyrotropic mode. Such coupling is present as otherwise the well-known frequency splitting of CW and CCW rotating spin-wave modes would not exist [20,21]. For vortex polarity up $(p=+1)$ the vortex gyromode only shows a $\mathrm{CCW}$ sense of rotation whereas for vortex core polarity down $(p=-1)$ only a $\mathrm{CW}$ sense of rotation is observed. This is also well known and can be calculated analytically using the Thiele equation [22,23]. As the spin-wave modes are coupled to the gyromode the response of the magnetization to $\mathrm{CW}$ or $\mathrm{CCW}$ excitation is different depending on whether the sense of rotation of the excited spin-wave mode is the same or opposite to the sense of rotation of the gyromode. Micromagnetic simulations in Fig. 4 reveal the differences in vortex dynamics for $\mathrm{CW}$ and CCW excitation (pulse length $T=60 \mathrm{ps)}$ which lead to the observed asymmetric switching thresholds. The vortex has a core pointing up (polarity $p=-1$ ) and a clockwise in-plane magnetization (circulation $c=-1$ ). Other combinations of $c, p$, and sense of the exciting fields are not discussed since they can be derived from the two cases shown here by simple symmetry considerations (see notes [24]). For the $\mathrm{CW}$ pulse sequence, mainly the $n=1, m=-1$ mode (eigenfrequency $f=7.1 \mathrm{GHz}$ ) is excited while for CCW excitation mainly the $n=1, m=+1$ mode (eigenfrequency $f=9.5 \mathrm{GHz}$ ) is excited. Due to the coupling with the vortex gyromode, excitation of the spin waves also leads to a motion of the vortex core. Both the simulated magnetization profile [Figs. 4(a) and 4(b)] and the trajectory of the vortex core [Figs. 4(c) and 4(d)] are clearly different leading to core reversal for $\mathrm{CCW}$ excitation only. The difference in switching
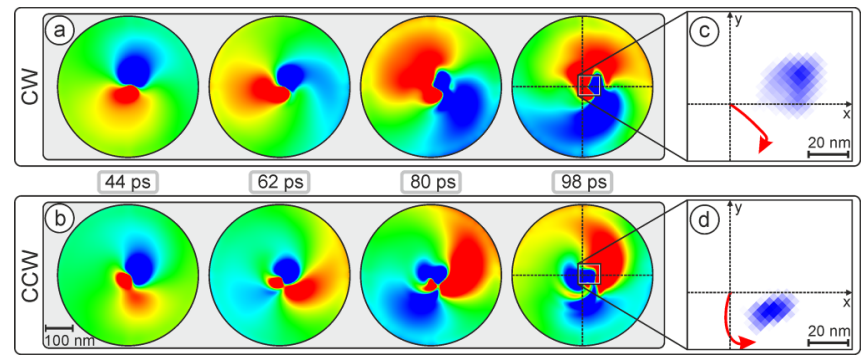

FIG. 4. (Color) Comparison of the simulated evolution of the outof-plane magnetization for the two rotation senses of the excitation. The different evolutions of the magnetization profiles for the $\mathrm{CW}$ and CCW case are displayed in the consecutive snapshots (a), (b) for an initial vortex core up (the CCW case corresponds to Fig. 2(a), but is not convolved with the experimental resolution here). The core trajectories are shown on the right side (c), (d) by the red arrows. Their origin corresponds to the equilibrium position of the core, $x=$ $y=0$. In the blue regions marked in (c), (d) the downwards oriented dip forms. These regions are obtained by averaging all cells with $M_{z}<-0.9 M_{S}$ over the sample thickness and from $t=0 \mathrm{ps}$ to $t=$ $100 \mathrm{ps}$. In the CCW case this dip region is closer to the original vortex core and more localized than in the $\mathrm{CW}$ case. Also, in the $\mathrm{CCW}$ case the core moves towards this dip region, while it moves away from it in the $\mathrm{CW}$ case leading to vortex core reversal for $\mathrm{CCW}$ excitation only.

threshold can be explained by these nonsymmetric vortex core trajectories. Guslienko et al. [25] explained the formation of the out-of-plane magnetized dip region preceding the vortex core inversion by the introduction of an effective gyrofield caused by the moving vortex core. In our case, the different core trajectories [Fig. 4(c) and 4(d)] lead to different gyrofields and therefore different dip formations. For both $\mathrm{CW}$ and $\mathrm{CCW}$ excitation the formation of the dip is located left of the vortex core movement [Fig. 4(c)]. But in the CCW case the dip forms closer to the core [Fig. 4(d)], and is more localized and partly enclosed by the core's trajectory. This results in core reversal where the dip is transformed into a vortex core pointing down while the original core is annihilated. In the CW case, the average distance between the created dip and the original vortex is larger and the dip is not confined by the core's trajectory. The dip partly splits up into a vortexantivortex (VA) pair followed by the annihilation of the original vortex core. However, this process does not occur across the whole thickness of the disk. In contrast, the splitting into a VA pair starts at the bottom layer of the three-dimensional simulation, and progresses only to approximately one third of the disk thickness. Subsequently, the remaining dip in the upper simulation layers dissolves, starting at the top layer. Thus the reversal process is not completed and the original vortex core up remains. This is in contrast to previous investigations $[4,5,7,8,13,14,19]$ where the formation of a VA pair led to core reversal.

\section{CONCLUSION}

In conclusion, we have experimentally demonstrated highspeed unidirectional vortex core reversal by orthogonal monopolar short pulses with pulse lengths between 90 and 
45 ps. We observe a unidirectional switching behavior in a broad range of field amplitudes and pulse lengths, in qualitative agreement with simulation. The asymmetry in the response to clockwise and counterclockwise excitation can be explained by the coupling between the gyrotropic mode and the spin waves resulting in a different evolution of the magnetization for opposite rotation senses. We imaged the spin wave and the vortex core evolution by time-resolved $\mathrm{x}$-ray microscopy in comparison with micromagnetic simulations, the results of which agree excellently with the experimental findings. As indicated in the x-ray movies and confirmed by the simulations a switching time of about 100 ps can be achieved. The possibility to further reduce the switching times for smaller sample diameters is explored by the corresponding micromagnetic simulations, where a lower, nearly size-independent limit of about $70 \mathrm{ps}$ is deduced. Situations are found where micromagnetic simulations have to be performed three dimensionally: A vortex-antivortex pair starts to form close to the original vortex core at the bottom of the disk without completing the formation across the whole thickness of the disk so that it dissolves later and does not lead to a switching of the original vortex core. Usually it is assumed that the creation of a vortex-antivortex pair occurs across the whole thickness and leads to a reversal of the original vortex core. In summary, these experimental and simulation studies provide important information in view of the use of a vortex core as a fast, stable, and low-power storage bit: The vortex core switching times can be reduced below $100 \mathrm{ps}$ and are thus in the order of the fastest electronically induced magnetization reversals in nanostructures [26] or even below. The unidirectionality covers a wide range of excitation amplitude and pulse duration and is therefore robust for arrays with irregular sample geometries. The ultimate value of switching times of about 70 ps cannot be reduced by decreasing the vortex size, whereby the optimum combination between size - determining the package density - and required field amplitude can be chosen for a given application.

\section{ACKNOWLEDGMENT}

We gratefully acknowledge the technical support by J. Baumann, M. Bechtel and B. Breimaier.
[1] E. Feldtkeller and H. Thomas, Phys. Kondens. Mater. 4, 8 (1965).

[2] T. Shinjo, T. Okuno, R. Hassdorf, K. Shigeto, and T. Ono, Science 289, 930 (2000).

[3] A. Wachowiak, J. Wiebe, M. Bode, O. Pietzsch, M. Morgenstern, and R. Wiesendanger, Science 298, 577 (2002).

[4] B. Van Waeyenberge, A. Puzic, H. Stoll, K. W. Chou, T. Tyliszczak, R. Hertel, M. Fähnle, H. Brückl, K. Rott, G. Reiss, I. Neudecker, D. Weiss, C. H. Back, and G. Schütz, Nature 444, 461 (2006).

[5] M. Weigand, B. Van Waeyenberge, A. Vansteenkiste, M. Curcic, V. Sackmann, H. Stoll, T. Tyliszczak, K. Kaznatcheev, D. Bertwistle, G. Woltersdorf, C. H. Back, and G. Schütz, Phys. Rev. Lett. 102, 077201 (2009).

[6] K. Yamada, S. Kasai, Y. Nakatani, K. Kobayashi, H. Kohno, A. Thiaville, and T. Ono, Nat. Mater. 6, 270 (2007).

[7] K. Yamada, S. Kasai, Y. Nakatani, K. Kobayashi, and T. Ono, Appl. Phys. Lett. 93, 152502 (2008).

[8] M. Curcic, B. Van Waeyenberge, A. Vansteenkiste, M. Weigand, V. Sackmann, H. Stoll, M. Fähnle, T. Tyliszczak, G. Woltersdorf, C. H. Back, and G. Schütz, Phys. Rev. Lett. 101, 197204 (2008).

[9] S.-K. Kim, K.-S. Lee, Y.-S. Yu, and Y.-S. Choi, Appl. Phys. Lett. 92, 022509 (2008).

[10] M. Curcic, H. Stoll, M. Weigand, V. Sackmann, P. Juellig, M. Kammerer, M. Noske, M. Sproll, B. Van Waeyenberge, A. Vansteenkiste, G. Woltersdorf, T. Tyliszczak, and G. Schütz, Phys. Status Solidi B 248, 2317 (2011).

[11] K.-S. Lee and S.-K. Kim, Phys. Rev. B 78, 014405 (2008).

[12] Y.-S. Yu, K.-S. Lee, H. Jung, Y.-S. Choi, M.-W. Yoo, D.-S. Han, M.-Y. Im, P. Fischer, and S.-K. Kim, Phys. Rev. B 83, 174429 (2011).
[13] M. Kammerer, M. Weigand, M. Curcic, M. Noske, M. Sproll, A Vansteenkiste, B. Van Waeyenberge, H. Stoll, G. Woltersdorf, C. H. Back, and G. Schuetz, Nat. Commun. 2, 279 (2011).

[14] M. Kammerer, H. Stoll, M. Noske, M. Sproll, M. Weigand, C. Illg, G. Woltersdorf, M. Fähnle, C. Back, and G. Schütz, Phys. Rev. B 86, 134426 (2012).

[15] M. Kammerer, M. Sproll, H. Stoll, M. Noske, M. Weigand, C. Illg, M. Fähnle, and G. Schütz, Appl. Phys. Lett. 102, 012404 (2013).

[16] G. Schütz, W. Wagner, W. Wilhelm, P. Kienle, R. Zeller, R. Frahm, and G. Materlik, Phys. Rev. Lett. 58, 737 (1987).

[17] M. J. Donahue and D. G. Porter, ооммF User's Guide, Version 1.0, Interagency Report No. NISTIR 6376 (National Institute of Standards and Technology, Gaithersburg, MD, 1999).

[18] A. Thiaville, J. M. García, R. Dittrich, J. Miltat, and T. Schrefl, Phys. Rev. B 67, 094410 (2003).

[19] R. Hertel, S. Gliga, M. Fähnle, and C. M. Schneider, Phys. Rev. Lett. 98, 117201 (2007).

[20] J. P. Park and P. A. Crowell, Phys. Rev. Lett. 95, 167201 (2005).

[21] K. Y. Guslienko, A. N. Slavin, V. Tiberkevich, and S.-K. Kim, Phys. Rev. Lett. 101, 247203 (2008).

[22] A. A. Thiele, Phys. Rev. Lett. 30, 230 (1973).

[23] D. Huber, J. Appl. Phys. 53, 1899 (1982).

[24] The response for a vortex with opposite circulation is obtained by rotating the magnetization by $180^{\circ}$ around the $z$ axis, mirroring at the $x-y$ plane, and flipping the direction of each macrospin. Consequently the cases for a vortex core down can be derived from the ones discussed: $p=+1$ and $\mathrm{CW}(\mathrm{CCW})$ excitation corresponds to $p=-1$ and $\mathrm{CCW}(\mathrm{CW})$ excitation.

[25] K. Y. Guslienko, K.-S. Lee, and S.-K. Kim, Phys. Rev. Lett. 100, 027203 (2008).

[26] O. J. Lee, D. C. Ralph, and R. A. Buhrman, Appl. Phys. Lett. 99, 102507 (2011). 